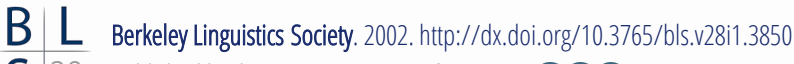

\section{A Lexical Approach to English Floating Quantifiers ${ }^{1}$}

\author{
EUN-JUNG YOO \\ Seoul National University
}

\section{Introduction}

This paper investigates how the syntactic and semantic characteristics of floating quantifiers (FQ) in English can be explained within the framework of the HeadDriven Phrase Structure Grammar. Previous analyses treat FQs either basically as NP quantifiers (Postal 1974, Maling 1976, Sportiche 1988, McCawley 1998), or as VP quantifiers that are syntactically and semantically distinct from NP quantifiers (Dowty \& Brodie 1984). In contrast, this paper proposes an analysis in which FQs are base-generated VP modifiers as in Dowty \& Brodie, but their logical (or semantic) contributions are made analogous to that of quantificational determiners, through a precise lexical description of FQs that specifies the information on "storage" and "retrieval" of the quantifier meaning. The proposed analysis is "lexical" in its nature in the sense that the syntactic and semantic properties concerning distribution and scope interaction are encoded as part of the lexical information that the FQs have.

\section{Properties of English FQ Constructions}

In English, only the quantifiers all, each, and both can "float." This contrasts to FQ constructions in languages like Japanese and Korean, which permit floating of a wider range of quantifiers including numeral quantifiers. Examples of English FQs are provided in (1) below.

(1) a. The children have all read the books.

b. The students have each arrived.

c. John's brothers have both read the book.

Floating quantifiers show characteristics that differ from normal quantificational determiners. First, FQs in English are subject-oriented. In (2), for

\footnotetext{
1 An earlier version of this paper was presented at the $15^{\text {th }}$ Pacific Asia Conference on Language, Information, and Computation in February 2001. I would like to thank both the BLS 28 audience and PACLIC 15 participants for their insightful comments. All remaining errors are my own.
} 


\section{Eun-Jung Yoo}

example, the quantifier can be construed with the subject NP, but not with the object NP.

(2) The children have all introduced their friends to us.

Thus (2) is not interpreted as 'The children have introduced all of their friends to us'. While some languages such as French, Japanese, and Korean allow quantifiers to "launch" from objects, no language is known to permit FQs hosted by adjuncts. This should be predicted in a proper analysis of FQs.

Second, FQs appear in front of a VP or AP.

(3) a. The computers all will have been moved to the new office.

b. The computers will all have been moved to the new office.

c. The computers will have all been moved to the new office.

d. The computers will have been all moved to the new office.

e. *The computers will have been moved all to the new office.

f. *The computers will have been moved to the new office all.

(4) a. We were all fast asleep.

(Quirk et al. 1985:382)

b. The children are all healthy.

Third, FQs are hosted by (more or less) definite plural NPs. The examples in (5) and (6), from Dowty \& Brodie, illustrate the kinds of NPs the FQ can semantically depend on.

(5) a. John, Mary and Susan all left.

b. John and Mary both left.

c. The students all left.

d. (?) Five students have all turned in their exams.

e. Five contestants, who were selected as finalists by the judge yesterday, will all perform again tomorrow.

(6) a. *John, Mary or Susan all left.

b. *Few students all left.

c. ${ }^{*}$ No students all left.

d.*At least five students all left.

NPs like five students usually have an interpretation equivalent to 'at least five students'. However, as Ladusaw (1982) and Dowty \& Brodie have suggested, they may also have an interpretation corresponding to 'exactly five particular students'. Example (5e) supports the view that in certain contexts, NPs like five students may have a definite interpretation. Thus when indefinite NPs have definite interpretations, they can be the semantic target a FQs.

Finally, unlike ordinary quantificational NPs, FQs do not exhibit scope 
ambiguity with respect to other scopal elements such as negation adverbs and modals (Dowty \& Brodie 1984:77). ${ }^{2}$

(7) a. The students all didn't leave.

$\mathrm{a}^{\prime} .\left[\forall \mathrm{x} \mid \operatorname{student}^{\prime}(\mathrm{x})\right]$ not $^{\prime}\left(\right.$ leave $\left.^{\prime}(\mathrm{x})\right)$

$\mathrm{b}$. The students didn't all leave.

$b^{\prime} \cdot \operatorname{not}^{\prime}\left(\left[\forall x \mid\right.\right.$ student $\left.^{\prime}(\mathrm{x})\right]$ leave' $\left.^{\prime}(\mathrm{x})\right)$

(8) a. The contestants all can win.

$a^{\prime} .\left[\forall x \mid\right.$ contestant $\left.^{\prime}(x)\right] \operatorname{can}^{\prime}\left(\right.$ win $\left.^{\prime}(x)\right)$

b. The contestants can all win.

$b^{\prime} \cdot \operatorname{can}^{\prime}\left(\left[\forall x \mid \operatorname{contestant}^{\prime}(\mathrm{x})\right]\right.$ win' $\left.^{\prime}(\mathrm{x})\right)$

With FQs, scope ambiguity is not observed in raising verb constructions either (McCawley 1998:631).

(9) a. His conclusions all appear to be incorrect.

b. His conclusions appear to all be incorrect.

(10) All his conclusions appear to be incorrect.

While (10) is ambiguous with regard to which takes wide scope, appear or all, in (9), the surface order between the two decides relative scope.

\section{Previous Analyses}

\subsection{Derivational Approaches}

Within transformational grammar, it is generally assumed that there is syntactic dependency between the host NP and the FQ. Thus the following FQ sentences in (11) are presumed to be related to the ones in (12) via movement.

(11) a. The children all left.

b. John believes the reporters both to have left.

(12) a. All (of) the children left.

b. John believes both (of) the reporters to have left.

There have been largely two approaches with respect to the syntactic dependency. The first is to posit rightward movement of the quantifier, deriving (11) from (12), as in Postal (1974), Mailing (1976), and McCawley (1998). As Haegeman \& Guéron (1999) note, however, such a downward derivation increases the overall

\footnotetext{
${ }^{2}$ Actualiy, the sentence ( $7 \mathrm{a}$ ) also has an additional reading in which negation takes wide scope. Following Dowty \& Brodie's suggestion, however, I will take this additional reading to be an outcome of metalinguistic negation.
} 
complexity of the grammar since the generalization can no longer hold that the moved constituent c-commands its trace. Another approach, which is proposed in Sportiche (1988), is to posit leftward movement of the NP, under the assumption that the phrase Q NP is in the [SPEC, VP] in the deep structure. While Sportiche mainly deals with French FQ constructions, the possibility of extending his analysis to English examples is also discussed. The sentences in (12) are derived, if the whole Q NP sequence is moved to [SPEC, IP], whereas the ones in (11) are generated when only the NP is moved to [SPEC, IP]. The latter option that derives (11) is described schematically in (13).

$$
\mathrm{NP}^{\wedge} \ldots\left[\mathrm{xn} \mathrm{Q}\left[\mathrm{NP}^{*} e\right] \mathrm{XP}\right]
$$

In (13), Q is adjacent to NP*, an NP trace, and there is an anaphoric relation between $\mathrm{NP}^{\wedge}$, the overt antecedent of $\mathrm{Q}$, and the trace. Thus the anaphor-like behavior between the host NP and Q is captured via movement.

However, as Sportiche himself notes, such an analysis raises a question for the examples where $\mathrm{Q}$ appears before the first auxiliary verb:

(14) a. The carpets will $\wedge^{\wedge}$ have ${ }^{\wedge}$ been ${ }^{\wedge}$ being ${ }^{\wedge}$ dusted for two hours.

b. The carpets all will have been being dusted for two hours.

In (14a), at least one empty NP position can be postulated in front of each verb, so that the occurrence of $Q$ in ${ }^{\wedge}$-marked positions is explained by (13). On the other hand, in (14b), the occurrence of all in front of the finite auxiliary verb is problematic, because there is no position available for the empty NP* due to the presence of the subject NP in [SPEC, I]. Sportiche claims that this problem can be solved if the trace is assumed to be in [SPEC, I] and the subject NP is topicalized. However, the assumption that the subject NP in (14b) is in the topic position is problematic, because topicalization of an object is still possible with an FQ in the pre-Infl position.

a. An office this large, the students all will desire.

b. To the opera, the students all have been.

Given that multiple topicalization is not allowed in English, examples like (15) show that the subject NP is not in the topic position in (14b).

Another problem with Sportiche's approach (and other derivational approaches) is that it cannot explain why English permits quantifier floating only for a few quantifiers (i.e., all, each, and both). It cannot be said that quantifier floating is allowed only for universal quantifiers, since every does not float. Given that some languages permit a wider range of quantifier floating (and that the characterization of the permitted range is not clear), this approach would need to introduce an ad hoc constraint to the grammar in order to prevent the derivation in (13) from applying to non-FQs in examples like (16). 
(16) a. *The students three will leave.

b. *The children most will leave.

Furthermore, as noted in Sportiche, this analysis provides no explanation as to why $F Q$ s do not appear before a $w h$-trace.
a. How angry do you think they all were
b. *How angry do you think they were all _-?

Since nothing prohibits the XP in (13) from being a wh-trace, Sportiche's analysis cannot account for the contrast in (17).

\subsection{Dowty \& Brodie's Non-Derivational Analysis}

Dowty \& Brodie (1984) propose that FQs are base-generated as VP modifiers. Thus in their analysis, FQs are introduced by the following syntactic rule:

$$
<1,[v P \text { Q VP], Q'(VP')>. }
$$

Their analysis focuses on providing formal semantics for FQ constructions. According to them, FQs, as VP quantifiers, belong to the semantic type distinct from that of determiners. More specifically, FQs map VP-denotations into argument NP denotations. Such a semantic function of FQs accounts for why quantifiers float only from arguments, but not from adjuncts. Furthermore, since the semantic rule for VP quantifiers depends on the non-empty intersection of all the sets in the NP denotation, it will require that the host NPs should be the class of definite plurals.

Dowty \& Brodie's analysis naturally explains the properties discussed in section 2, without positing any transformation process. However, since it relies heavily on rule-to-rule semantic interpretations, it is hard to be incorporated into a syntactic framework that does not assume a compositional model-theoretic semantics that accompanies each expression. In what follows, I will present an alternative non-derivational analysis of FQ constructions, in which both syntactic and semantic aspects of these sentences can be accounted for via interactions with each other within a feature structure.

\section{FQs and Quantifier Retrieval}

\subsection{Lexical Representations of FQs}

In presenting an analysis of FQs, we must first consider what kind of logical forms can be assigned to the sentences with FQs. Since the host NPs are plural NPs, how to deal with their semantic representation should be decided. Following Link (1983), I assume that collective and mass entities are contained in the model as individuals. 
Link (1983) and Dowty (1986) argue that the addition of all to plural NPs invokes universal quantificational force, and has an effect dubbed as the Maximizing Effect, requiring the predicate in question to be true of every member of the group. Incorporating this generalization, the logical form of (19a) can be expressed by (19b), using an informal restricted quantificational logic notation:

a. The students all sneezed.

b. [the $y \mid$ students' $\left.^{\prime}(\mathrm{y})\right]\left([\forall \mathrm{x} \mid\right.$ constituent-of' $\left.(\mathrm{x}, \mathrm{y})]\left(\operatorname{sneezed}^{\prime}(\mathrm{x})\right)\right)$

In (19b), 'const(ituent)-of' is a function that resembles Link's (1983) relation 'atomic-part-of', and identifies each member $x$ of the group $y$ which is contextually salient.

On the other hand, in the case of a collective predicate, it is semantically abnormal that the predicate holds for each member of the given group. Accordingly, the group entity itself should be predicated.

(20) a. The students all gathered.

b. [the $y \mid$ students' $\left.^{\prime}(y)\right]\left(\left[\exists x \mid\right.\right.$ group $^{\prime}(x) \&([\forall z \mid$ constituent-of' $(z, y)]$ (constituent-of' $(\mathrm{z}, \mathrm{x})))]($ gathered' $(\mathrm{x})))$

The logical representation (20b) may look complicated. However, such complexity seems to be unavoidable in order to take into account the group reading in examples like (21).

(21) Most of the 20 students gathered.

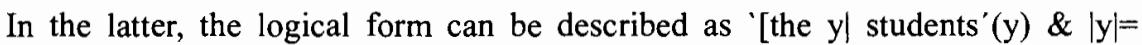
$20]\left(\left[\exists x \mid\right.\right.$ group $^{\prime}(\mathrm{x}) \&$ ([most $\mathrm{z} \mid$ constituent-of' $\left.(\mathrm{z}, \mathrm{y})\right]($ constituent-of' $\left.\left.(\mathrm{z}, \mathrm{x}))\right)\right]$ (gathered'(x)))'.

As (19b) and (20b) indicate, the logical representations of FQ sentences vary depending on the distributive/collective interpretation of the predicate. Furthermore, when the FQ all is used with a collective predicate, an existential quantifier arises in the interpretation. We take this fact to support a lexical approach to the FQ construction because, in such approach, two different lexical descriptions of the FQ may result in different logical interpretations.

Now let us consider how the informal logical representation discussed so far can be expressed in our theory. Our representation of quantifier scope is based on that of Pollard \& Sag (1994), in which the semantic contribution of a word or phrase is represented as the value of the CONT(ENT) attribute in the feature structure. In the CONT value of a type psoa (parameterized-state-of-affairs), the quantifier in the QUANT(IFIER)S list is taken to have scope over the

${ }^{3}$ I thank an anonymous abstract reviewer of the HPSG 2001 for pointing this out to me. 
NUC(LEUS) value. Accordingly, the CONT of (19a) can be described by (22). When there is more than one quantifier in the QUANTS, the preceding member is taken to have scope over the preceded one(s).
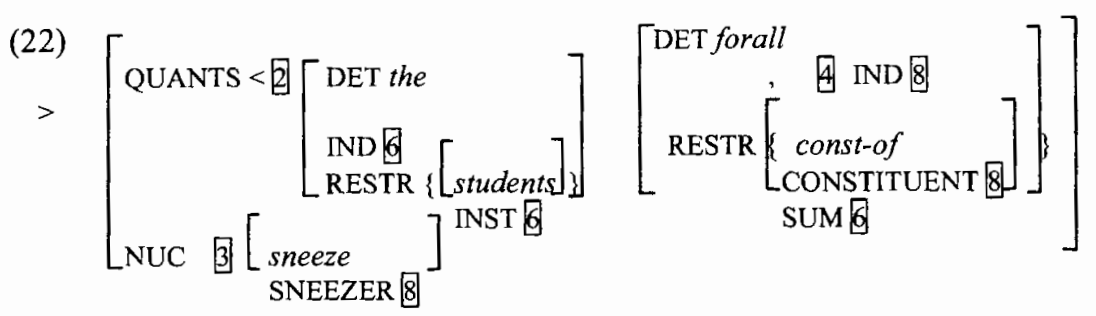

In (22), the numeral tag 6 indicates a plural entity, while 8 expresses a constituent member of the plural entity. Likewise, based on (20b), the CONT of (20a) can be described as a structure whose QUANTS contain a definite quantifier followed by an existential quantifier, and whose NUC(LEUS) consist of the psoa gather.

In Pollard \& Sag's 1994 theory of quantifier scope, the meaning of a quantifier 'starts out in storage' in the QS(TORE) and is "inherited" into a larger phrase in the structure, and then "retrieved" to take scope over a certain phrase or sentence. This theory is revised and extended in Pollard \& Yoo (1998) in order to account for scope phenomena in raising verb constructions and unbounded dependency constructions. It contains a set of new assumptions for the account of quantifier scope: i) the QSTORE feature is relocated as a LOCAL attribute, ii) a new feature POOL is introduced as an additional LOCAL attribute, iii) "ordinary" lexical heads "collect" all the QSTORE values of their "selected arguments," iv) QSTORE values are inherited only from the semantic daughter of a phrase, and v) quantifier retrieval is possible either at a lexical head or a phrase. Among the QSTORE, POOL, and RET(RIEVED) values, the following constraint holds:

(23) For a sign, the RETRIEVED value is a list whose set of elements forms a subset $\mathrm{S}$ of the POOL value; and the QSTORE value is the relative complement of the set $\mathrm{S}$.

As in Pollard \& Sag, the elements in the RETRIEVED also appear in the QUANTS value to take their scope.

When considering how to represent the FQ all in the lexicon, the most natural assumption that we can make is that all introduces a quantifier in its POOL, just like quantificational determiners. In Pollard \& Yoo, the words that give rise to a quantifier meaning are classified as quant(ifier)-word, and their POOL and QSTORE values are lexically specified. These words are distinguished from ordinary lexical heads in that their QSTORE values are not the union of all the QSTORE values of their arguments.

What I will further propose in this paper is that the RETRIEVED value of 
certain quantifier-introducing words should be lexically specified as well. Thus, I claim that in addition to ordinary cases where a quantifier is retrieved at some structural node, obeying a set of constraints on the features POOL, QS(TORE), RET(RIEVED), and QUANTS, it is necessary to specify the RET (and thus QUANTS) values lexically for some quantifier-introducing words. ${ }^{4}$

\subsection{Analysis and Explanation}

Based on the foregoing discussion, I propose that the quantifier scoping in English FQ sentences can be accounted for by specifying retrieved quantifiers in the lexical entry of the FQ all. As discussed in 3.1, the quantifiers arising in the FQ sentences vary depending on the semantic type of the predicates. Accordingly, two lexical entries are provided for the FQ all. The first entry in (24) is for the sentences with a distributive predicate.

$$
\begin{aligned}
& \text { all } l_{1} \text { (for distributive predicates) }
\end{aligned}
$$

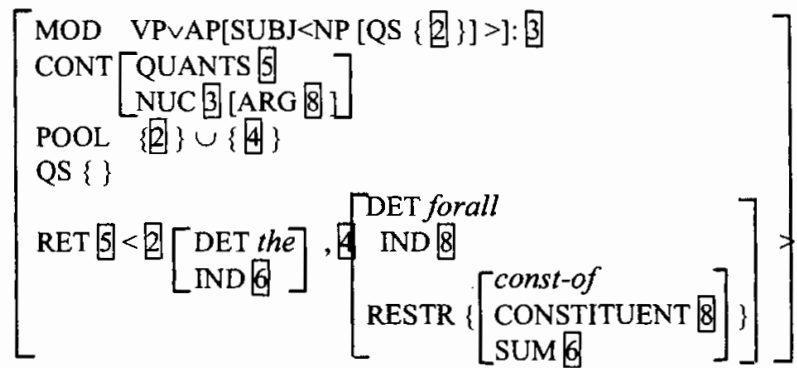

In (24), the QUANTS list, which has the same value 5 as the RET, contains two quantifiers, 2 and 4 . The quantifier 2 indicates a definite quantifier arising from the subject NP, and 4 corresponds to ' $[\forall x \mid$ constituent-of' $(x, y)]$.' When the FQ appears in a sentence, the quantifiers will scope over the NUC(LEUS) value, which is the CONT of the VP. Accordingly, (19a) will have the CONT in (22).

The second lexical entry of the FQ all for collective predicates is described in (25).

${ }^{4}$ Lexical specification of quantifier retrieval is independently motivated, because there are other examples like (i) where a quantifier word needs to contain a nonempty QUANTS value.

(i) Every kid's favorite toy broke.

See Yoo (2001) for the detailed discussion of how the narrow scope reading ' $[$ the $x \mid$ toy' $(x) \&$ $\left(\left[\forall y \mid \operatorname{kid}^{\prime}(y)\right]\right.$ poss' $\left.\left.(y, x)\right)\right]$ broke'( $\left.x\right)^{\prime}$ can be accounted for by the lexical specification of retrieved quantifiers in the lexical entry of the possessive determiner 's. 
all 2 (for collective predicates)

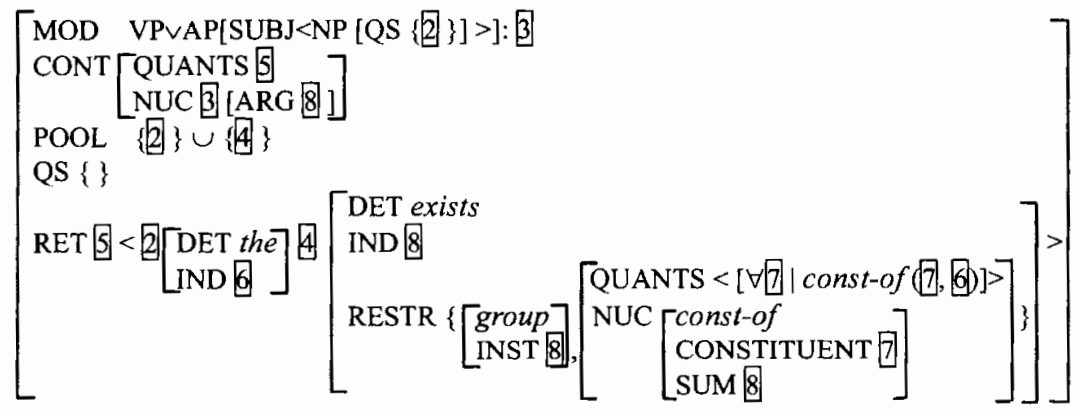

I assume that the choice between (24) and (25) is made on semantic grounds. Thus, to analyze collective reading examples like (20a) with the entry (24) will yield semantic anomaly, because it is not the case that each constituent member of the group 'gathers'. Given the lexical entry in (24), the sentence in (19a) can be assigned the structure in (26). In (26), the numeral tags 2 and 4 indicate the elements in the QUANTS in (22) and the tag 3 corresponds to the NUC in (22).

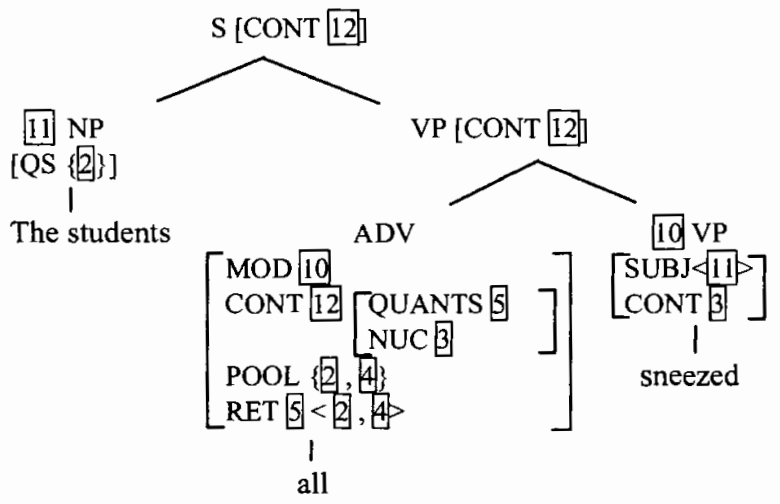

Now let us consider how the lexical entries in (24) and (25) provide explanations for the properties of FQs discussed in section 2. First of all, the fact that only three words, all, each, and both, are used as FQs is easily explained, since only these three will have a lexical entry that looks like (24) or (25). Other quantifiers in English will only have the entry of determiners.

Second, the subject-oriented property of FQs is accounted for, since the index of the quantifier 8 is related to that of the VP or AP subject, i.e. 6 , via the relation constituent-of. This analysis also can be extended to account for FQs in the languages allowing both subjects and objects to have associated FQs. This is because the FQ may access the arguments of the verb that it modifies via the 
ARG(UMENT)-ST(RUCTURE) feature (or the SUBJ and COMPS features) of the verb.

Third, since all selects a VP or AP via its MOD feature, it appears as a modifier of a VP or AP in the structure. In other words, all, as an adjunct daughter, combines with a VP or AP, constituting a head-adjunct phrase. Yet it remains to be answered why the modifier does not appear in the post-VP positions, as ( $3 \mathrm{e}-\mathrm{f}$ ) illustrate. There seem to be two ways to account for this. One solution is to posit a Linear Precedence (LP) rule that holds between "light" modifiers like FQs and a modified VP/AP. (See Abeille \& Godard (1999:92) for the same type of LP rule proposed for French.) In the LP rule, we can specify that a "light" non-head daughter must precede the head daughter. Altematively, if we adopt Bouma et al.'s (2001) proposal that post verbal adjuncts are added to the DEPENDENTS list by a lexical rule, then we can specify that certain adverbs such as all, both, each, only, never, certainly, and probably are not added by this lexical rule. The only way these adverbs are introduced in a sentence is via a head-adjunct structure in which the head comes after the adjunct.

Moreover, as Sag \& Fodor (1994) argue, if FQs are treated as VP-adjoined modifiers, then the ungrammaticality of $(17 \mathrm{~b})$ can be elegantly explained by employing a traceless approach to extraction available in HPSG. Examples like (17b) are ruled out, simply because there is no VP/AP that FQs can combine with.

Next, the generalization that FQs are hosted by (contextually) definite NPs is accounted for, because, according to (24) and (25), the SUBJ element of the VP that all modifies has a QS member whose DET is the. Therefore, if the host NP is an indefinite NP like some students, the DET value of the NP's QS is exists, which conflicts with the constraint imposed on the descriptions (24) and (25).

Furthermore, the use of the relation constituent-of predicts that a singular entity cannot be the host of a FQ. Since there is no plural (or group) entity from which its constituents can be extracted, the following examples are correctly ruled out:

a. *The student has all/each arrived.

b. ${ }^{*}$ Each student had all arrived.

Finally, in (24) and (25), the universal quantifier associated with the FQ is required to be retrieved lexically and take scope over the VP that it modifies. Thus the quantifier associated with all does not inherit into a larger phrase or the lexical head that selects the phrase. Consequently, when the modified VP contains a modal or negation, all has wide scope over such scopal elements. This explains the interpretations of (7a) and (8a). Likewise, when all is lower than the modal or negation element in the structure, as in (7b) and (8b), it takes narrow scope.

So far our discussion has been focused on the quantifier all. The analysis proposed for all can be easily extended to the discussion of each or both. As Dowty \& Brodie note, unlike all, each and both are restricted to individual-level NPs and do not have group reference. 
(28) a. *Each (of the) student(s) gathered in the stadium.

b. ${ }^{*}$ Both students are a happy couple.

This implies that, between the two types of logical forms, (19b) and (20b), only the (19b) type is available. The distinction between each and both will, of course, come from the fact that both is limited to an NP whose cardinality is 2. Since the FQ each does not occur with collective predicates, its lexical entry will look like the first entry of all.

The present analysis can also account for the examples like (29)-(30) where the host NP contains a quantifier with a more complex RESTR value.

(29) The three students have all played tennis.

(30) John's students all came to the party.

In (29), we take the quantifier in the QS(TORE) value of the subject NP to be '[the $y \mid$ students' $(y) \&|y|=3]$,' in which $|y|$ indicates the cardinality of $y$. As (24) imposes, this quantifier, indicated by 2 in (24), cannot be retrieved or inherited into a larger VP. Instead, it is retrieved lexically, together with the universal quantifier arising from all. Consequently, in (29), there will be two quantifiers in the QUANTS list of the sentence, i.e., '[the $\left.y\left|\operatorname{students'}^{\prime}(y) \&\right| y \mid=3\right]$ ' and ' $[\forall x \mid$ constituent-of'(x,y)]'.

Likewise, in (30), the quantifier in the RET and QUANTS list of all is the definite quantifier associated with the subject NP and the universal quantifier arising from all. Thus the two quantifiers in the QUANTS list of the sentence will be '[the $y \mid$ students' $(y)$ \& possess' (john, y)]' and '[ $\forall \mathrm{x} \mid$ constituent-of' $(\mathrm{x}, \mathrm{y})]]$.' Accordingly, complicated examples such as (29-30) can also be handled by the lexical entries in (24) and (25).

\section{Concluding Remarks}

In this paper, a constraint-based, lexical approach to English floating quantifier constructions has been presented. Drawing on Dowty \& Brodie's assumption that FQs are base-generated as VP modifiers, I have focused on providing an appropriate CONTENT value for the sentences containing FQs. In representing the semantic contribution of the FQs, I have proposed that both "quantifier storage" and "quantifier retrieval" take place lexically at their sites. Accordingly, a FQ, which functions as a semantic head of a VP, carries all the necessary semantic information for the VP, including the quantifier meaning. With such a mechanism of lexical specification of quantifier retrieval, I have shown that various properties of FQs, with respect to syntactic distributions, the types of host NPs, and scope interaction with adjacent elements, can be accounted for by a precise description of the lexical entry of the FQs. 


\section{References}

Abeille, A. and D. Godard. 1999. A lexical approach to quantifier floating in French. In G. Webelhuth et al. (eds.) Lexical and Constructional Aspects of Linguistic Explanation, 81-96. Stanford, CA: CSLI.

Bouma, G. and R. Malouf. \& I.A. Sag. 2001. Satisfying constraints on extraction and adjunction. Natural Language and Linguistic Theory 19: 1-65.

Dowty, D. 1986. Collective predicates, distributive predicates, and all. ESCOL 86: 97-115.

Dowty, D. and B. Brodie. 1984. The semantics of "floated" quantifiers in a transformationless grammar. In WCCFL 3, 75-90. Stanford, CA: CSLI.

Haegemann, L. and J. Guéron. 1999. English Grammar. Blackwell Publishers.

Ladusaw, W. 1982. Semantic constraints on the English partitive construction. In WCCFL 1, 231-242.

Link, G. 1983. The logical analysis of plurals and mass terms: a lattice-theoretic approach. In R. Bauerle et al. Meaning, Use, and Interpretation of Language. Berlin and New York: Walter de Gruyter.

Maling, J. M. 1976. Notes on quantifier postposing. Linguistic Inquiry 7: 708-718. McCawley, J. D. 1998. The Syntactic Phenomena of English. Chicago: The University of Chicago Press.

Pollard, C. and I. A. Sag. 1994. Head-Driven Phrase Structure Grammar. Chicago: The University of Chicago Press.

Pollard, C. and E. J. Yoo. 1998. A unified theory of scope for quantifiers and whphrases. Journal of Linguistics 34: 415-445.

Postal, P. M. 1974. On Raising. Cambridge: MIT Press.

Quirk, R., S. Greenbaum, G. Leech, \& J. Startvik. 1985. A Comprehensive Grammar of the English Language. London: Longman.

Sag, I. A. and J. D. Fodor. 1994. Extraction without traces. In WCCFL 13. Stanford, CA: CSLI.

Sportiche, D. 1988. A theory of floating quantifiers and its corollaries for constituent structure. Linguistic Inquiry 19: 425-449.

Yoo, E.-J. 2001. English floating quantifiers and lexical specification of quantifier retrieval. Language and Information 5:1-15. Seoul, Korea.

Eun-Jung Yoo

Department of English Language and Literature

Seoul National University

Seoul, 151-742, Korea

ejyoo@snu.ac.kr 